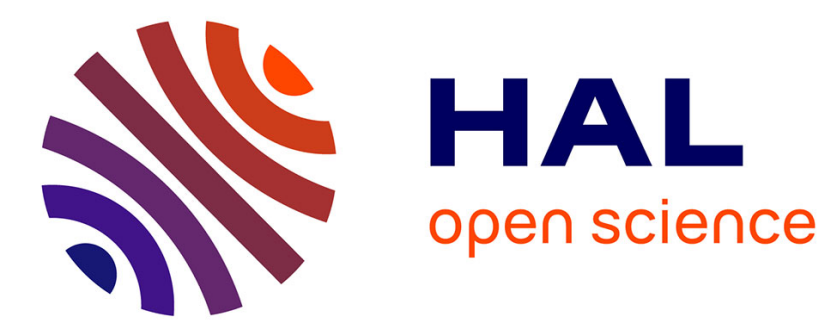

\title{
Geometry Analysis of Superconducting Cables For The Optimization of Global Performances
}

Nicolas Lermé, Petr Dokládal

\section{To cite this version:}

Nicolas Lermé, Petr Dokládal. Geometry Analysis of Superconducting Cables For The Optimization of Global Performances. ICPRAM, Feb 2016, Rome, Italy. hal-01237380v2

\section{HAL Id: hal-01237380 \\ https://hal.science/hal-01237380v2}

Submitted on 18 Dec 2015

HAL is a multi-disciplinary open access archive for the deposit and dissemination of scientific research documents, whether they are published or not. The documents may come from teaching and research institutions in France or abroad, or from public or private research centers.
L'archive ouverte pluridisciplinaire HAL, est destinée au dépôt et à la diffusion de documents scientifiques de niveau recherche, publiés ou non, émanant des établissements d'enseignement et de recherche français ou étrangers, des laboratoires publics ou privés. 


\title{
Geometry Analysis of Superconducting Cables for the Optimization of Global Performances
}

\author{
Nicolas Lermé ${ }^{1,2 *}$, Petr Dokládal ${ }^{1}$ \\ ${ }^{1}$ MINES Paris Tech, PSL Research University, Centre for Mathematical Morphology, Fontainebleau, France \\ ${ }^{2}$ Autonomous Systems Group, Université Paris Sud, Gif-sur-Yvette, France \\ email: nicolas.lerme@u-psud.fr, petr.dokladal@mines-paristech.fr \\ Keywords: \\ Segmentation, Registration, Shape analysis, Clustering, Superconductivity, Cable.
}

Abstract:

Superconducting cables have now become a mature technology for energy transport, high-field magnets (MRI, LHC) and fusion applications (ToreSupra, and eventually ITER and DEMO). The superconductors are extremely brittle and suffer from electrical damages brought by mechanical strain induced by electromagnetic field that they generate. An optimal wiring architecture, obtained by simulation, can limit these damages. However, the simulation is a complex process and needs validation. This validation is performed on real $3 \mathrm{D}$ samples by the means of image processing. Within this objective, this paper is, to our best knowledge, the first one to present a method to segment the samples of three types of cables as well as a shape and geometry analysis. Preliminary results are encouraging and intended to be later compared to the simulation results.

\section{INTRODUCTION}

\subsection{Motivation and scope}

Superconducting cables have now become a mature technology in energy transport, high-field magnets (medicine (MRI), high-energy physics (LHC)) and magnetic confinement in fusion applications (ToreSupra, eventually ITER, DEMO).

A superconducting cable presents a multiscale internal structure. Such a cable consists of strands arranged together given some application-dependent architecture. Individual strands are composite structures either formed by superconducting microfilaments embedded in a metallic matrix or by a thin superconducting layer deposited onto a metallic substrate.

A substantial drawback of some of these cables is the fragility of the superconductors. Mechanical strains can indeed cause deformations, thus degrading their performance. Whereas these strains can be limited during shaping (wiring or winding) or thermal cool-down, they remain problematic during operation when exposed to high electromagnetic fields (due to its own Lorentz force, particularly under cyclic loading).

However, the performance degradation can be diminished by optimizing the geometry of the ca-

${ }^{*}$ This work was funded by the ANR project ANRGUI-AAP-05 (2013-2017) and performed during the post-doctoral research project of Nicolas Lermé at the Centre for Mathematical Morphology. bles. Optimize the performance of these cables is thus essential and could directly benefit to a large number of research and industrial actors. The global performances of the cable are simulated using models of the electrical and/or mechanical behavior of the cable structure (Torre et al., 2014; Manil et al., 2012). Given the complexity of various types of cables, the validation of these models is done by statistical comparison of the geometry of the models to the geometry of real cables obtained from tomography images. Depending on the result of these comparisons, the design of the cables can be optimized, until a better cable architecture is obtained.

This paper focuses on the identification of the experimental geometry on three types of cables, involving mostly ${ }^{2}$ automatic registration, segmentation, clustering and features extraction tasks. To our best knowledge, this is the first paper providing methods and results for the geometry analysis on these cables. For each type of cable, we provide below the physical parameters and the image characteristics.

\subsection{Available cables}

Three distinct types of cables are considered with different architectures, appearances and composition: the cables-in-conduit (Weiss et al., 2007),

\footnotetext{
${ }^{2}$ The segmentation of cables-in-conduit require initial manual markers.
} 

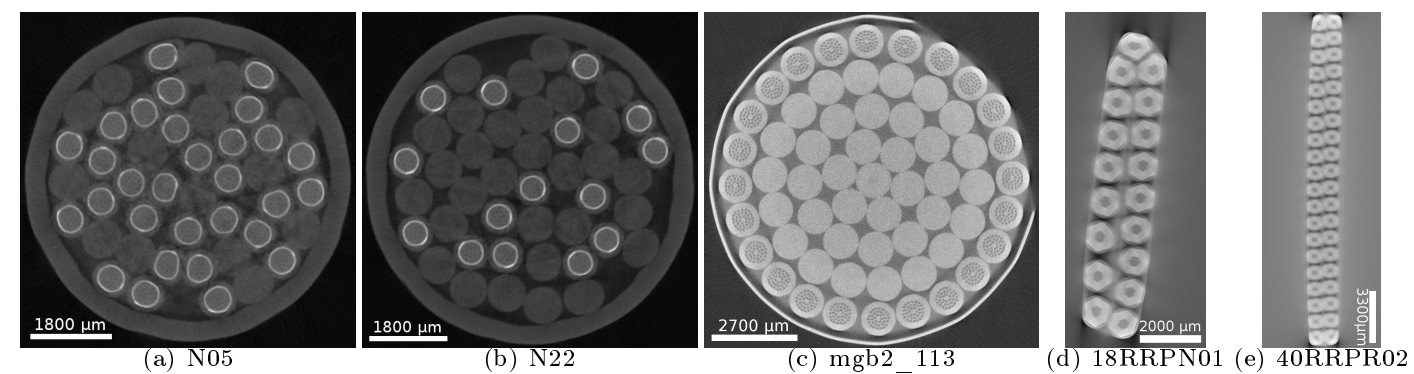

Figure 1: Cross-sectional images from cables-in-conduit (a,b), power cables (c) and Rutherford cables (d,e).

the Rutherford cables (Oberli, 2013; Milanese et al., 2012) and the power cables (Seidel and Sturge, 2009; IEC, 2004). Several samples of each with different parameters were analyzed (see Tab. 1 and 2 for their characteristics, and Fig. 1 to assess the variety and the quality of the images).

Cables-in-conduit (see Fig. 1(a,b)) consist of superconducting strands (s.c.) and nonsuperconducting strands (n.s.c.). S.c. strands are composed of kernels in $\mathrm{Nb}_{3} \mathrm{Sn}$ and bronze (white) wrapped by a jacket in copper (gray) while n.s.c. strands are made of copper (gray). All strands are twisted together in a multistage fashion according to a predefined cable pattern composed of stages and petals. For instance, the pattern $(3,3,5)$ of the cable N05 has three stages. The first (lower) stage consists of twisted triplets (15 petals), each with 2 s.c. and 1 n.s.c. strands. These triplets are again twisted by three ( 5 petals) and finally by five ( 1 petal), thus leading to a total of 30 s.c. and 15 n.s.c. strands. All strands are then inserted in a stainless steel conduit. Some void fraction is kept to enable the circulation of a cooling fluid (helium). Notice that all stages are twisted with a different twist-pitch. Typically used in fusion applications, these cables can transport currents of $45 \mathrm{kA}$ under a magnetic field of $12.3 \mathrm{~T}$, exposed to transversal Lorentz forces of $554 \mathrm{kN} / \mathrm{m}$.

Power cables (see Fig. 1(c)) consist of s.c. strands in $\mathrm{MgB}_{2} /$ nickel alloy and n.s.c. copper strands. All strands are arranged in concentric layers, twisted and inserted in a corrugated cryogenic envelope. Again, some void fraction is kept to enable the circulation of a coolant (gaseous and eventually liquid helium). S.c. strands are located on the outer layer. Typically used in energy distribution, each strand can convey a current of $400 \mathrm{~A}$ under a magnetic field of $1 \mathrm{~T}$, developing radial, centripetal, Laplace forces of $400 \mathrm{~N} / \mathrm{m}$.

Rutherford cables (see Fig. 1(d,e)) roughly consist of s.c. strands composed of cores in copper (gray) surrounded by an intermediary zone containing $\mathrm{Nb}_{3} \mathrm{Sn}$ filaments (white), themselves wrapped in a copper jacket (gray). All strands are twisted to form a two-layers flat cable, compressed to a well-controlled rectangular section. Depending on the design used, the filaments zone is arranged to form either an hexagon or a circle. Typically used in medical imaging and highfield magnets, these cables can transport currents of the order of $20 \mathrm{kA}$ under a magnetic field of $10-15 \mathrm{~T}$, exposed to Lorentz forces of $1-5 \mathrm{MN} / \mathrm{m}$.

\subsection{Outline of the paper}

The rest of this paper is as follows. In Section 2, we briefly explain the registration of overlapping samples. In Section 3, we detail, for each type of cable, the proposed methods for extracting the structures of interest from the resulting images. In Section 4, we propose indicators reflecting potential damages. Finally, we present results in Section 5 and discuss future work in Section 6.

\section{IMAGE REGISTRATION}

The cable samples being too long to be imaged at once, multiple overlapping scans have been acquired with a constant translation step along the z-axis ${ }^{3}$. Each scan has then been registered on the previous one by minimizing the mean square error of the difference of intensities over the overlapping region along the z-axis. The minimization along the z-axis is sufficient since no residual displacement along the $x$ and $y$ axes was observed.

\section{IMAGE SEGMENTATION}

The purpose of the segmentation is to extract the contours of strands (and the conduit, if need be) and their centerlines. Most structures to extract are nearly circular and touch each other. Their appearance and contrast however differ for all cable architectures. Distinct algorithms have been designed for all types of cables with their own set of parameters. Some of these parameters still

\footnotetext{
${ }^{3}$ From here, the $z$-axis will refer to the longitudinal one while $x, y$ axes will refer to transversal ones.
} 
Table 1: Image characteristics (top) and physical parameters (bottom) of the cables-in-conduit (left to the double bar) and power cables (right to the double bar).

\begin{tabular}{|c|c|c|c|c|c|}
\hline & N05 & $\mathrm{N} 22$ & $\mathrm{~N} 25$ & mgb2 113 & mgb2 133 \\
\hline Image size $(x, y, z)$ & $\begin{array}{c}750 \times 800 \times \\
12431\end{array}$ & $\begin{array}{c}750 \times 850 \times \\
12424\end{array}$ & $\begin{array}{c}750 \times \\
750 \times 7745\end{array}$ & $\begin{array}{c}1200 \times \overline{1200 \times} \\
5972\end{array}$ & $\begin{array}{c}1300 \times \overline{1605 \times} \\
6071\end{array}$ \\
\hline Resolution $(\mu \mathbf{m})$ & 12 & 12 & 12 & 10 & 10 \\
\hline Memory usage (GB) & 6.94 & 7.37 & 4.05 & 8.1 & 12 \\
\hline Number of s.c./n.s.c. (all) strands & $30 / 15(45)$ & $15 / 30(45)$ & $30 / 15(45)$ & $24 / 37(61)$ & $24 / 37(61)$ \\
\hline Strand $\oslash(\mu \mathbf{m})$ & 800 & 800 & 800 & 1130 & 1330 \\
\hline Avail. sample length $(\mu \mathbf{m})$ & 149172 & 149088 & 92940 & 59270 & 60710 \\
\hline Twist-pitch sequence ( $\mathrm{mm})$ & $45 / 85 / 125$ & $45 / 85 / 125$ & $35 / 65 / 110$ & 227 & 260 \\
\hline First triplet (s.c.,n.s.c.) & $(2,1)$ & $(1,2)$ & $(2,1)$ & 7 & 7 \\
\hline Cable pattern & $(3,3,5)$ & $(3,3,5)$ & $(3,3,5)$ & / & / \\
\hline Void fraction (\%) & 25 & 33 & 33 & 24.7 & 24.7 \\
\hline
\end{tabular}

Table 2: Image characteristics (top) and physical parameters (bottom) of the Rutherford cables.

\begin{tabular}{|c|c|c|c|c|c|c|c|}
\hline & COP-RRP & I8PTTNO1 & 18PTTNO1_2 & 18RRPNOI & 18RRPNO1_2 & 40RRPRण & 40RRPRण2 \\
\hline Image size $(x, y, z)$ & $\begin{array}{c}1500 \times \\
300 \times 1300\end{array}$ & $\begin{array}{c}750 \times \\
1100 \times 1200\end{array}$ & $\begin{array}{c}1600 \times 500 \times \\
1200\end{array}$ & $\begin{array}{c}1100 \times \\
750 \times 1200\end{array}$ & $\begin{array}{c}1700 \times 700 \times \bar{x} \\
1200\end{array}$ & $\begin{array}{c}1600 \times \\
500 \times 1200\end{array}$ & $\begin{array}{c}1500 \times \\
500 \times 1200\end{array}$ \\
\hline Resolution $(\mu \mathbf{m})$ & 15 & 10 & 6.75 & 10 & 6 & 15 & 15 \\
\hline Memory usage (MB) & 558 & 945 & 916 & 945 & 1434 & 916 & 859 \\
\hline Number of strands & 40 & 18 & 18 & 18 & 18 & 40 & 40 \\
\hline Strand $\oslash(\mu \mathbf{m})$ & 1050 & 1020 & 1000 & 1050 & 1046 & 1080 & 1035 \\
\hline $\begin{array}{l}\text { Avail. Sample } \\
\text { Length }(\mu \mathrm{m})\end{array}$ & 19500 & 12000 & 8100 & 12000 & 7200 & 18000 & 18000 \\
\hline
\end{tabular}

require a manual tuning (in this case, it will be mentioned below). However, most of them appear to be stable and expressed with respect to the image resolution and cables characteristics.

These algorithms share common difficulties to overcome to get reliable measurements. First, they must be able to assess slight deviations from the circularity, be robust to noise, artifacts and poor contrast. Second, they must be fast and able to handle large volume data (possibly more than shown in Tab. 1 and 2). To accommodate these constraints, a simple approach has been preferred (whenever possible) where the $3 \mathrm{D}$ volume is segmented as a sequence of $2 \mathrm{D}$ images along the z-axis. This allows to run some steps in parallel. Once strand contours have been obtained, the centerlines consist of the strand centroids. Moreover, we assume the following known and constant: the diameter of a strand (denoted by $S_{d r}$ ), the thickness of the strand jacket (denoted by $S_{j t}$ ), the thickness of the conduit of the cable (denoted by $C_{c t}$ ) as well as the number of s.c. and n.s.c. strands (denoted by $N_{s c}$ and $N_{n s c}$, resp.).

\subsection{Rutherford cables}

The core of strands being better contrasted than the jackets, we propose an automatic procedure that relies on the filaments zone to extract the contours of strands in two steps (see Fig. 2).

\subsubsection{Extraction of cores}

First, an Alternate Sequential Filter (ASF) is applied on the source image with a squared structuring element up to the size of $0.03 \times S_{d r}$ (Sternberg, 1986) (image $I_{D}(\mathrm{a})$ ). This allows us to both denoise the image and fill the interstices between filaments. Next, a filling algorithm is applied on the image $I_{D}$ (image $I_{F}(\mathrm{~b})$ ). The image $I_{F}$ is then subtracted from the image $I_{D}$ (image $I_{A}(\mathrm{c})$ ). Once the image $I_{A}$ thresholded (image $\left.I_{T}(\mathrm{~d})\right)$, it both contains cores and interstices. To discard the interstices, a mask is built by thresholding the image $I_{F}$ (image $I_{T^{\prime}}(\mathrm{e})$ ) and applying a morphological erosion with a square of size $0.15 \times S_{d r}$ as structuring element (image $I_{M}(\mathrm{f})$ ). Finally, the mask $I_{M}$ is intersected with the image $I_{T}$ and the $3 \mathrm{D} N_{s c}$ largest connected components are kept. The resulting components are labeled and correspond to the cores (image $I_{C}(\mathrm{~g})$ ). Due to the variety of images, notice that the above thresholds need to be manually adjusted, independently of ground truths (see Section 5.1.1).

\subsubsection{Extraction of strands}

As shown in Fig. 2, the filaments zone is poorly contrasted and strands touch with each other. To overcome these difficulties, we introduce several priors (near circularity, small displacement and volume conservation of strands sections) and proceed in two successive steps.

First, a distance transform is computed from each labeled core of $I_{C}$ using an efficient pixel queue algorithm (Ikonen, 2005). This algorithm has a worst-case complexity of $O(n \log n)$ (where $n$ is the number of pixels in the image). Each distance map is computed using the WDTOCS metric, described in (Ikonen, 2005). Let $I: \Omega \subset$ $\mathbb{Z}^{2} \rightarrow \mathbb{R}$ be an image and $\mathcal{N}$ be a neighborhood. Without loss of generality, we propose to use

$$
\mathcal{N}=\{(p, q) \in(\Omega \times \Omega) \mid\|p-q\| \leq \sqrt{2}\},
$$




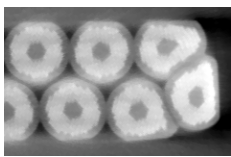

(a) $I_{D}$

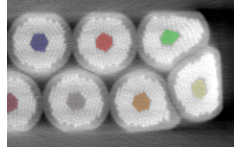

(g) $I_{C}$

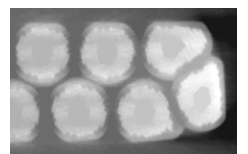

(b) $I_{F}$

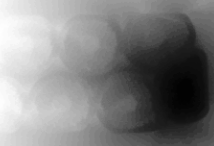

(h) $I_{D M}\left(\alpha=10^{4}\right)$

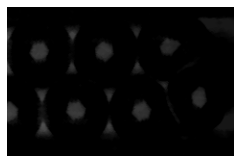

(c) $I_{A}$

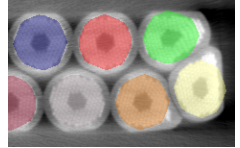

(i) $I_{R}(\alpha=0)$

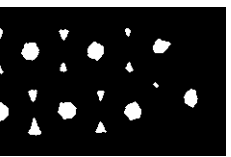

(d) $I_{T}$

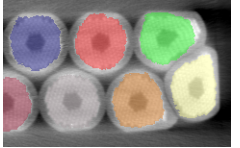

(j) $I_{R}\left(\alpha=10^{4}\right)$

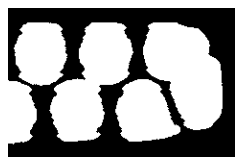

(e) $I_{T^{\prime}}$

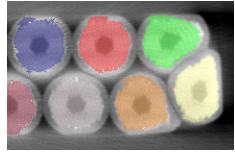

(k) $I_{R}\left(\alpha=10^{6}\right)$

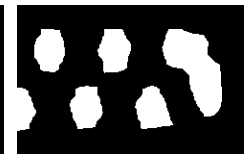

(f) $I_{M}$

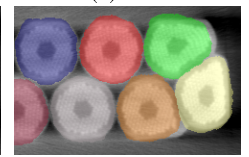

(1) $I_{R^{\prime}}$

Figure 2: Steps for extracting the cores (a-g) and the strands (h-l) from an image of a Rutherford cable (COPRRP). The source image is first denoised by an ASF (a). Once (a) filled (b), it is subtracted from (a), giving (c). An intermediate result (d) is obtained by thresholding (c). A mask (f) is built by thresholding (b) (giving (e)) and applying a morphological erosion on the result (f). (f) is then intersected with (d) and the 3D $N_{s c}$ largest connected components are kept. The resulting components are labeled and correspond to the cores (g). A region-growing algorithm is then used to extract the filaments zone, based on geodesic distance maps whose amount of regularity is controlled by a parameter $\alpha(\mathrm{h})$. The impact of $\alpha$ after region-growing is shown when it is small (i), moderate (j), large (k). The strands are obtained by expanding the filaments zone with $\alpha=0$ (l).

where $\|\cdot\|$ is the $L^{2}$ norm in $\mathbb{Z}^{2}$. For any pair $(p, q) \in \mathcal{N}$, the WDTOCS metric is defined as

$$
\operatorname{dist}(p, q)=\sqrt{\|p-q\|^{2}+\alpha\left(I_{p}-I_{q}\right)^{2}},
$$

where $\alpha \in \mathbb{R}^{+}$is a parameter that balance geometric and image information. The larger the parameter $\alpha$ is, the more the image information is taken into account. An example of distance map from the rightmost core is shown in (h) where the distance is proportional to the intensity (the dynamic of the image has been stretched for visualization purpose). Distance transforms being independent from each other, their computation is in practice performed in parallel using OpenMP. Once all the distance maps obtained using $\alpha>0$, a region-growing algorithm is applied to obtain the outer contour of the filaments zones: all cores grow simultaneously by greedily selecting the pixels having the minimum cost based on their respective distance maps, until a target volume is reached ${ }^{4}$. The effect of varying the parameter $\alpha$ after region-growing is depicted on the image $I_{R}$ when $\alpha$ is small (i), moderate $(\mathrm{j})$ and large $(\mathrm{k})$. Finally, the contours of strands are obtained by expanding the filaments zones in such a way that none of them is favored. This is achieved by repeating the above steps for $\alpha=0$ (image $I_{R^{\prime}}(1)$ ). In our experiments, we set $\alpha=10^{4}$.

\subsection{Cables-in-conduit}

As shown in Fig. 3, the s.c. strands are better contrasted than the n.s.c. ones. Based on this observation, we propose a three-steps scheme that first extracts automatically the conduit and then

\footnotetext{
${ }^{4} \mathrm{It}$ is adjusted if the filaments zone is an hexagon.
}

the s.c. strands. The n.s.c. strands are then semiautomatically extracted based on initial markers.

\subsubsection{Extraction of the conduit}

First, the source image is denoised by an ASF with a squared structuring element up to the size of $0.05 \times S_{d r}$ (Sternberg, 1986) (image $I_{D}(\mathrm{a})$ ). To further flatten homogeneous areas, the image $I_{D}$ is filtered by a Gaussian of size $0.05 \times S_{d r}$ and thresholded (image $I_{T}(\mathrm{~b})$ ). Next, a morphological opening, a holes filling algorithm and then a morphological closing are applied on the image $I_{T}$ using a square of size $0.2 \times S_{d r}$ as structuring element (image $I_{M}(\mathrm{c})$ ). Assuming the ideal conduit is nearly circular, an ellipse is fitted on the image $I_{M}$. Its size is subtracted by $2 \times C_{c t}$ to fit the inner contour of the conduit (image $I_{R}(\mathrm{~d})$ ).

\subsubsection{Extraction of the s.c. strands}

The strategy employed here is to rely on kernels to extract the contours of strands. For doing so, the source image is first thresholded (image $I_{T^{\prime}}$ (e)). Next, the resulting contours are closed using a morphological dilation with a square of size $0.25 \times S_{j t}$ as structuring element and holes are filled (image $I_{F}(\mathrm{f})$ ). To properly align the segmentation on the contours of kernels, a watershed algorithm (Meyer, 1991) is applied on the source image (image $I_{W}(\mathrm{~g})$ ). The outside marker is the complementary of the image $I_{F}$ and the markers representing the kernels are obtained by performing a morphological erosion of the image $I_{F}$ with a squared structuring element of size $1.25 \times S_{j t}$. As for Rutherford cables, s.c. strands are finally obtained by expanding the kernels from the image 


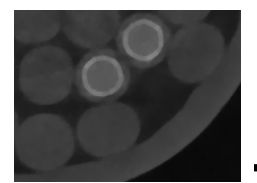

(a) $I_{D}$

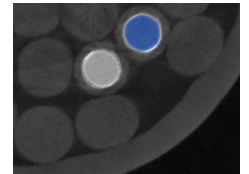

(g) $I_{W}$

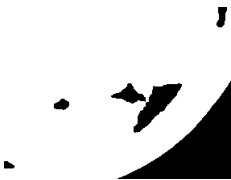

(b) $I_{T}$

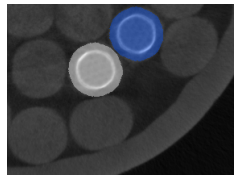

(h) $I_{R^{\prime}}$

J

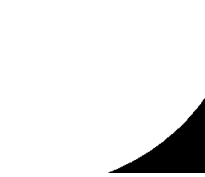

(c) $I_{M}$

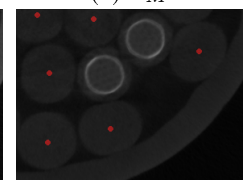

(i) $I_{C}$

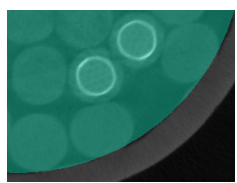

(d) $I_{R}$

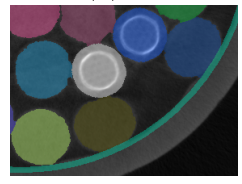

(j) $I_{R^{\prime \prime}}(z=0)$

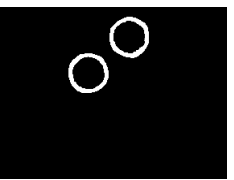

(e) $I_{T^{\prime}}$

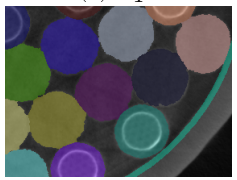

k) $I_{R^{\prime \prime}}(z=6211)(1) I_{R^{\prime \prime}}(z=12423)$

Figure 3: Steps for segmenting the conduit (a-d), the s.c. strands (e-h) and the n.s.c. strands (i-l) from an image of a cable-in-conduit (N22). The source image is first denoised by an ASF (a). Next, (a) is filtered by a Gaussian and thresholded (b). Holes are filled in (b) by various morphological operations (c). An ellipse is then fitted on (c) and adjusted on the inner contour of the conduit (d). Once the conduit obtained, (a) is thresholded from the source image (e), dilated and filled (f). Using (d) and (f), kernels are obtained by applying a watershed algorithm (g) on the source image. S.c. strands $(\mathrm{h})$ are then extracted by expanding (g) (same approach as Rutherford cables with $\alpha=0)$ and subtracting the complement of (d). Finally, n.s.c. strands are extracted with $\alpha>0(\mathrm{j}-1)$, constrained by $(\mathrm{d})$ and $(\mathrm{h})$ but from the strands centroids $\mathrm{f}$ the previous image (i).

$I_{W}$ with $\alpha=0$ (see Section 3.1.2) and subtracting the complement of the mask $I_{R}$ (image $I_{R^{\prime}}(\mathrm{h})$ ).

\subsubsection{Extraction of the n.s.c. strands}

To overcome the poor contrast on n.s.c. strands, we adopt the same strategy as for Rutherford cables (see Section 3.1.2) but with two differences. First, the geodesic distances are computed using $\alpha>0$ but constrained in $I_{R^{\prime}} \backslash I_{R}$. Second, the centroids of s.c. strands must be provided as initialization (image $I_{C}(\mathrm{i})$ ). Geodesic distance maps are computed from these centroids and the region-growing algorithm is applied. Once the centroids computed on the resulting contours of strands, the same procedure is applied on the next image (image $I_{R^{\prime \prime}}$ ). This process continues until the end of the cable is reached. To illustrate the correctness of the propagation, the image $I_{R^{\prime \prime}}$ is shown with the n.s.c. strands obtained at the beginning $(\mathrm{j})$, the middle $(\mathrm{k})$ and the end (l) of the cable. Unlike Rutherford cables, it is important to notice that such an approach can fail to recover the contours of n.s.c. strands since centroids depend on the result of the previous image. Such a situation can occur when the contribution of the right term in Eq. 1 is insufficient. In that case, the procedure becomes unable to stick to the contours of strands. A suitable value of the parameter $\alpha$ must therefore be chosen carefully. In our experiments, we have chosen to set $\alpha=8 \times 10^{4}$.

\subsection{Power cables}

For extracting strands, a convenient solution would be to use the same approach as for
Rutherford cables (see Section 3.1.2) and for the n.s.c. strands of the cables-in-conduit (see Section 3.2.3). As shown in Fig. 1, despite the important amount of noise, the images of power cables present however a much better contrast compared to the images of the other types of cables. We detail below a simple procedure that automatically find the contours of the conduit and then the strands, both assumed to be nearly circular.

First, the source image is thresholded (image $\left.I_{T}(\mathrm{a})\right)$ and holes are filled (image $I_{F}(\mathrm{~b})$ ). From the image $I_{F}$, the largest connected component is discarded and a morphological opening is applied with a squared structuring element of size $0.1 \times$ $C_{c t}$ (image $\left.I_{O}(\mathrm{c})\right)$. An ellipse is then fitted on the image $I_{O}$. Its size is subtracted by $C_{c t}$ to fit the inner contour of the conduit (image $I_{R}(\mathrm{~d})$ ).

Second, the source image is denoised by an ASF with a squared structuring element up to the size of $0.05 \times S_{d r}$ (image $I_{D}(\mathrm{e})$ ) (Sternberg, 1986). A first rough estimate of the contours of strands is obtained by thresholding the image $I_{D}$ (image $\left.I_{T^{\prime}}(\mathrm{f})\right)$. The complement of the mask $I_{R}$ is then subtracted from the image $I_{T^{\prime}}$ and a morphological opening is applied with a square of size $0.03 \times S_{d r}$ as structuring element (image $I_{I}$ (g)). Next, 3D connected components that do not spread all along the cable in the background are removed (image $I_{H}(\mathrm{~h})$ ). From the image $I_{H}$, the Euclidean distance to the contours is computed and the resulting image is filtered by a Gaussian of size $0.25 \times S_{d r}$ (image $I_{D M}$ (i)) to ensure a good detection of maxima (image $I_{M}(\mathrm{j})$ ). Finally, the contours of strands are obtained by applying a watershed (Meyer, 1991) performed on 


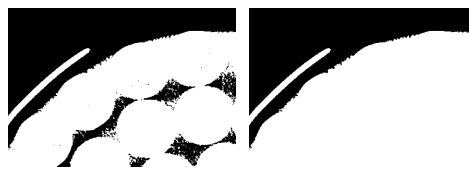

(a) $I_{T}$

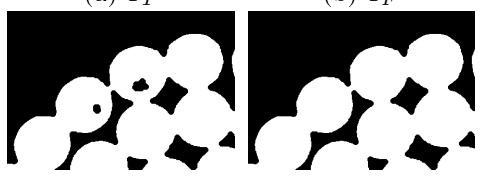

(g) $I_{I}$

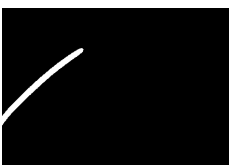

(c) $I_{O}$

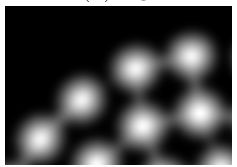

(i) $I_{D M}$

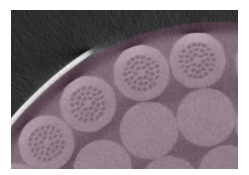

(d) $I_{R}$

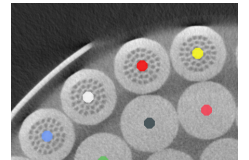

(j) $I_{M}$

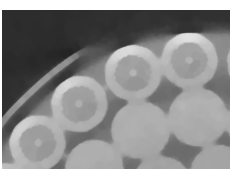

(e) $I_{D}$

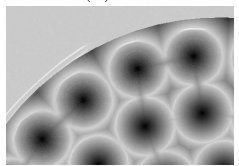

(k) $I_{S}$

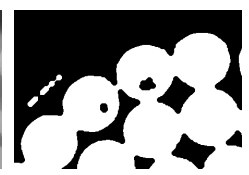

(f) $I_{T^{\prime}}$

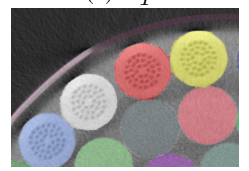

(1) $I_{R^{\prime}}$

Figure 4: Steps for segmenting the conduit (a-d) and the strands (i-1) of a power cable (mgb2 113). First, the source image is thresholded (a) and filled (b). From (b), the largest connected component is discarded and a morphological opening is applied (c). An ellipse is fitted on (c) and adjusted on the inner contour of the conduit (d). Second, the source image is denoised by an ASF (e) and thresholded (f). The complement of (d) is subtracted from (f) and a morphological opening is applied $(\mathrm{g})$. 3D connected components that do not spread all along the cable are removed $(\mathrm{h})$. The Euclidean distance to the contours of $(\mathrm{h})$ is computed, filtered by a Gaussian (i) and maxima are identified (j). Strands contours are obtained by applying a watershed (l) on an image (k) combining the morphological gradient of the source image and the inverted distance map to ideal contours from maxima.

the summed image $I_{S}(\mathrm{k})$ composed of the inverted distance map to ideal contours from maxima and the morphological gradient (multiplied by a factor set to 0.1 ) of the source image (image $I_{R^{\prime}}(\mathrm{l})$ ). The multiplier ensures the strands sections to remain mostly circular along the cable.

\section{DAMAGE INDICATORS}

During operation, the cables can be exposed to transversal Lorentz forces up to several $\mathrm{MN} / \mathrm{m}$. These forces induce deformation and/or damages to strands that come out as various geometrical features. The features detailed below aim at detecting these deformations.

\subsection{Strand Section Compression}

Any deviation to the circularity of strands is a potential source of damage that need to be measured. A variety of shape descriptors has been proposed in the literature. In this setting, desirable properties of shape descriptors are the invariance to translation and rotation, the robustness to noise and a reasonable sensitivity. The papers (Montero, 2009; Žunić, 2012) gather a large number of descriptors, out of which two have been selected as applicable in this context and offering similar performance. Let $S \subset \mathbb{Z}^{2}$ be a strand section of a $2 \mathrm{D}$ binary image taken along the z-axis. These two descriptors are defined as follows:

$$
E F(S)=\frac{\lambda_{\max }}{\lambda_{\min }} \in[1,+\infty[\text { (Elongation Factor), }
$$

where $\lambda_{\min }, \lambda_{\max }$ are the eigenvalues of the covariance matrix of the strand section $S$, and

$\left.\left.C M P N(S)=\frac{(\sharp S)^{2}}{2 \pi\left(\mu_{2,0}+\mu_{0,2}\right)} \in\right] 0,1\right]$ (Compactness), where $\sharp$ denotes the cardinality of a set and $\mu_{p, q}$ denotes the moment of order $(p+q)$ of the strand section $S$. EF and CMPN are statistical descriptors. EF is lower-bounded by one for a circle and increases for elongated shapes while CMPN is upper-bounded by one for a circle and tends to zero for elongated shapes. These two descriptors verify the above mentioned properties.

\subsection{Curvature}

For all the considered types of cables, strands can locally bend significantly and these locations are potentially source of damage. Do detect bendings with a large amplitude, we propose to measure the local curvature of the strand centerlines.

The curvature measures a failure of a curve to be straight. It is is positive or null and equal to the inverse of the radius of the tangent circle.

Let us represent a centerline with a two times continuously differentiable space curve $\mathcal{C}(t)=$ $(x(t), y(t), z(t))^{T} \in \mathbb{R}^{3}$, parameterized by $t$. Also, we denote resp. by $\gamma^{\prime}(t)$ and $\gamma^{\prime \prime}(t)$ the first and second and derivatives of $\mathcal{C}$ with respect to $t$. The local curvature of the curve $\mathcal{C}$ is defined by

$$
\kappa(t)=\frac{\left\|\gamma^{\prime}(t) \times \gamma^{\prime \prime}(t)\right\|}{\left\|\gamma^{\prime}(t)\right\|^{3}}
$$

where $\|$.$\| and \times$ are respectively the $L^{2}$ norm and the cross product, both in $\mathbb{R}^{3}$. In what follows, we briefly discuss some numerical considerations. First, to avoid division by zero, Eq. 2 is set to zero when the denominator is smaller than some $\varepsilon>0{ }^{5}$. Second, the derivatives are approximated by standard finite central differences. Due to the 
presence of noise, estimating small values of curvatures is however a delicate problem. Nevertheless, under the assumption that the strands cannot mechanically bend over some limit value, the finite differences are computed using a grid spacing (denoted by $\Delta h$ ) proportional to the strand diameter $S_{d r}$. A large value of $\Delta h$ allow us to assess small curvature values despite the noise. Also, we choose not to consider extremities of centerlines. Finally, to yet increase the robustness, the centerlines are first smoothed by a Gaussian filter of standard deviation $\sigma=0.05 \times S_{d r}$.

\subsection{Void fraction}

It is the ratio of area not occupied by the strands over the area delimited by the inner part of the conduit. A large void fraction means that strands are likely to move and bend. Once the cables are segmented, it can be obtained without difficulty.

\subsection{Twist-pitch}

The twist-pitch refers to the stranding periodicity of a cable. Unlike the power cables or the Rutherford cables, the cables-in-conduit are wired and compacted so that some (limited though) randomness is injected in the architecture. As a consequence, the estimation of twist-pitches only applies to cables-in-conduit. Given the presence of some quantity of randomness, we will estimate the twist-pitches using the autocorrelation of the strand centerlines. Recall that different stages are wired with different twist-pitches (see Tab. 1). The estimation of these twist-pitches thus implies to identify the stages of the cable.

The identification of the petals at different stages can be seen as a hierarchical clustering problem, constrained by the pattern of the cable. To form clusters at a given stage, a possible choice is to use pairwise distances of all strands. Indeed, closely running strands are more likely to belong to the same petal.

Let us formalize the above problem. We denote the set of $N$ centerlines of length $K$ by $\left\{c_{i}\right\}_{i=1}^{N}$, where $c_{i} \in \mathbb{R}^{3 K}$. For any couple $(i, j) \in$ $\{1, \ldots, N\}^{2}$, we define $d\left(c_{i}, c_{j}\right)$ as the distances between $c_{i}$ and $c_{j}$. The distance is different depending of the norm. A reasonable choice for $d$ is the mean distance, based on the $L^{2}$ norm. Additionally, for a given number of stages (denoted by $M$ ), we denote by $P_{m}$ the number of petals, for any $m \in\{1, \ldots, M\}$. We also denote by $\left\{\varphi_{m}\right\}_{m=1}^{M}$ a set of applications where $\varphi_{m}:\{1, \ldots, N\} \rightarrow\left\{1, \ldots, P_{m}\right\}$ assigns a label to each centerline of the stage $m$, for any $m \in\{1, \ldots, M\}$. Last, we denote by $\mathbf{1}_{\{.\}}$the indicator function returning 1 if its argument is true, 0 otherwise. Then, we propose to solve the constrained hierarchical clustering problem by finding a minimizer to

$$
\sum_{m=1}^{M} \sum_{(i, j) \in\{1, \ldots, N\}^{2}} \frac{\mathbf{1}_{\left\{\varphi_{m}(i)=\varphi_{m}(j)\right\}} d\left(c_{i}, c_{j}\right)}{2 P_{m} K M},
$$

subject to the following constraints:

1. $\left\{\varphi_{m}\right\}_{m=1}^{M}$ is a hierarchy,

2. For each petal at $M=1, N_{s c} / N_{n s c}$ are fixed,

3. For any stage, the size of each petal is fixed.

For a single stage, Eq. 3 can be put under the form of an integer linear program (with a number of variables and constraints both of $O\left(N^{2} P_{1}\right)$ ) and solved exactly using an integer linear programming solver. For this experiment, the last version of CPLEX has been chosen for its good performances (Mittelmann, 2007). Even for a simplistic situation where a single stage and a limited number of centerlines are considered $(N=15)$, several days of calculus are needed. This remains acceptable for this setting but becomes intractable for large cables with hundreds of strands.

To overcome this situation, we use a greedy strategy for solving Eq. 3 heuristically. An illustrative example is provided in Fig. 5 for the N05 cable (clusterings are superimposed on source images). For the first stage, random triplets satisfying the above second and third constraints, are formed (see Fig. 5(a)). A greedy heuristic is then applied, that consist in swapping the pairs of centerlines satisfying the above second constraint and making the strongest decrease of Eq. 3. This process is iterated until no swaps can be performed (see Fig. 5(b)). The next stages are optimized in the same way, except that (i) the initialization is based on the clustering obtained at the previous stage and (ii) that pairs of groups of centerlines are swapped instead of centerlines (see Fig. 5(c,d)). This allows us to keep clusterings as a hierarchy (first above constraint). Finally, the overall approach is run 100 times and the solution having Eq. 3 minimum is kept.

\subsection{Free areas and segments}

Subject to an intense magnetic field, strands of cables-in-conduit will transversally move wherever there is locally an insufficient compaction. Identifying these locations is therefore an important indicator of fragility of some particular stranding pattern. For doing so, we need a measure to estimate the area where a strand can 


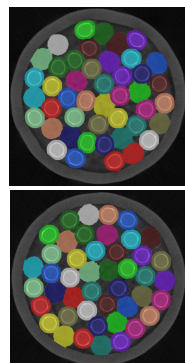

(a)

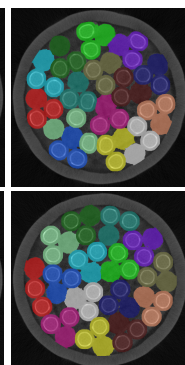

(b)

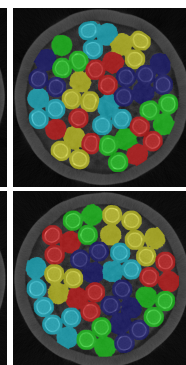

(c)

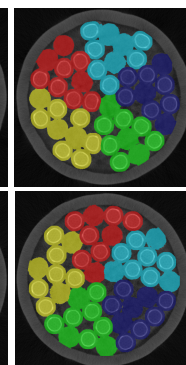

(d)
Figure 5: Example of constrained hierarchical clustering for identifying the the stages of the cable-inconduit N05 at the beginning (top row) and the end (bottom row). (a): initialization of first stage, (b): result - first stage petals (triplets), (c): initialization of second stage, (d): result - second stage petals.

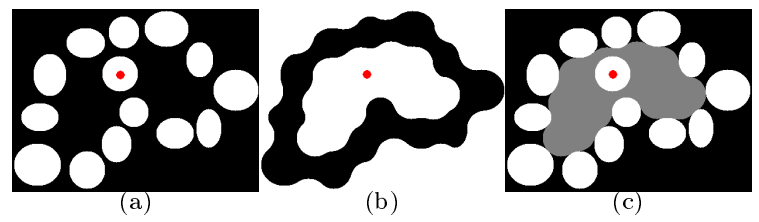

Figure 6: Toy example for estimating the area where a strand section $(S$, red dot) can freely move with respect its neighboring ones $(T)$. (b): complement of the morphological closing of $T$ by $S$, (c): geodesic reconstruction (light gray) of $S$ under (b).

move. Let us denote by $S \subset \mathbb{Z}^{2}$ the section of a particular strand and by $T$ that of the union of the remaining ones, both from the same $2 \mathrm{D}$ binary image taken along the z-axis (see Fig. 6(a)). We denote by $F$ the area where $S$ can move, obtained by applying a morphological closing on $T$ using $S$ as a structuring element (denoted by $\varphi_{S}(T)$, see Fig. 6(b)) and then performing a geodesic reconstruction of $S$ under the complement (denoted by $(.)^{c}$ ) of $\varphi_{S}(T)$ (see Fig. 6(c)):

$$
F=\left[\delta_{B}(S) \wedge\left[\varphi_{S}(T)\right]^{c}\right]^{\infty},
$$

where $\delta_{B}(S)$ denotes the dilation of $S$ by a ball $B$ of unit radius, $\wedge$ denotes the logical AND and [.] $]^{\infty}$ denotes iterated until idempotence (achieved in practice, in a finite number of iterations). Based on $F$, we propose a first possible descriptor, Free Transversal Area (FTA), expressed as

$$
\operatorname{FTA}(F, S)=\frac{\sharp(F \backslash S)}{\sharp S} \times 100 \in \mathbb{R}^{+} .
$$

The above descriptor is null when the strand cannot move and increases as the strand gets a larger space to move. The distribution of FTA is a descriptor revealing a fragility of a cable exposed to a strong magnetic field. Extracting connected components where FTA is greater than some positive value alongside a strand permits to identify portions of strands able to move transversally. This allows us to propose a second descriptor of cable fragility, Free Transversal Segments (FTS), defined as the set of all lengths of such segments.

\section{EXPERIMENTAL RESULTS}

\subsection{Validation}

\subsubsection{Segmentation}

Due to the variety of used cables and the variable quality of images, the segmentation is a delicate task needing validation. We propose to validate the results by relying on an expert. First, this expert did a visual check of the segmented cables to ensure there are no inconsistencies. Second, the contours of the strands and the conduit (where applicable) have been manually delineated by this expert on a few 2D images, equally spaced along the z-axis. For cables-in-conduit and power cables, one image every $1.5 \mathrm{~mm}$ and $8.5 \mathrm{~mm}$ has been selected, resp. Due to the variety of resolution, three images have been only selected per Rutherford cable.

To assess the accuracy of the results, we use evaluation metrics on the contours of strands and the conduit using the Volumetric Overlap Error (VOE), the Relative Absolute Volume Difference (RAVD), the Root Mean Square Distance (RMSD) and the Maximum Distance (MD) (see (Ginneken et al., 2007)) but also the popular Dice Coefficient (Dice, 1945). Although the metrics from (Ginneken et al., 2007) were presented in a clinical setting, we do believe that they still remain relevant here. In addition, we also propose to compare the position of centerlines using the $L^{2}$ norm between centroids (CD).

The results of these comparisons are summarized in Tab. 3. For each metric and type of cable, the mean and standard deviation are provided. For cables-in-conduit, the negative value of the mean RVD for s.c. and n.s.c. strands indicates that their volume is under-estimated and suggest an adjustment of segmentation parameters. As expected, the error on n.s.c. strands appears to be larger than of the s.c. ones due to their poorly contrasted contours (e.g. a factor of two for the mean CD). For most of the metrics used, compared to other types of cables, the worst error is reached for Rutherford cables. Nevertheless, all metrics globally show that the structures of interest (especially the conduit) are well segmented with, for instance, a mean DC greater than $94 \%$ and a mean RMSD always less than 3 pixels. The 

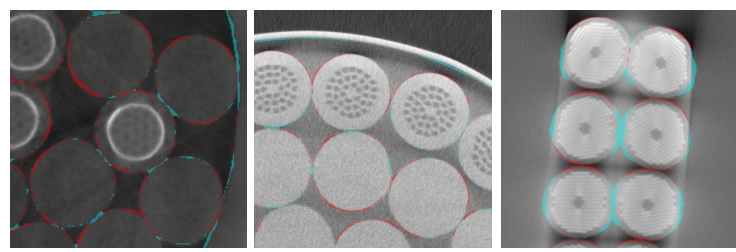

N22 (96.42\%)
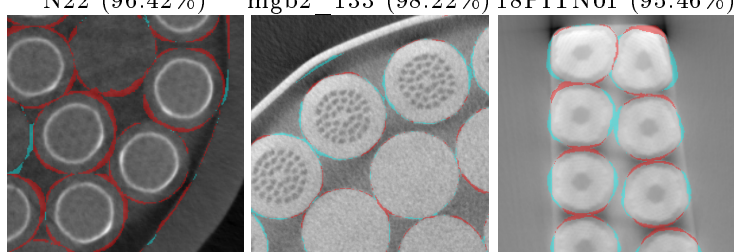

N05 (95\%) mgb2_113 (97.78\%) 40RRPR01 (93.4\%)

Figure 7: Accuracy of the segmentation procedures on the contours strands and the conduit (if available). For each type of cable, we select the 2D images from the obtained segmentations and the manual ones with the same position along the z-axis, having the mean largest (top row) and smallest (bottom row) DC. Both images are combined to show false negatives (red) and false positives (cyan), superimposed on the corresponding source image. For each image, the average $\mathrm{DC}$ is provided between parentheses.

position of centerlines is also well preserved with a mean CD always less than 1.4 pixels.

A subset of these results is illustrated in Fig. 7. For each type of cable, we provide the $2 \mathrm{D}$ images from the obtained segmentations and the manual ones with the same position along the z-axis (where manual segmentations are available) having the largest (top row) and the smallest (bottom row) average DC. Each couple of images is then combined to show false negatives (red) / false positives (cyan), superimposed on the source image.

\subsubsection{Damage indicators}

To validate the capacity of the strand sections compression indicators (see Section 4.1) to detect non-circularities, we propose the following experiment (see Fig. 8). Two distinct locations have been identified along the same strand of the N25 cable, where the section is either ellipsoid or circular. It has been verified that the compression is unique along the strand. The contours of these sections superimposed on source images are shown on top row. On the bottom row are shown the response returned by the indicators along the cable. Both EF and CMPN depict a good robustness to noise and highlight well the compression by reaching a large peak at the compressed location and remaining close to zero or one elsewhere. The same observations were made at other loca-

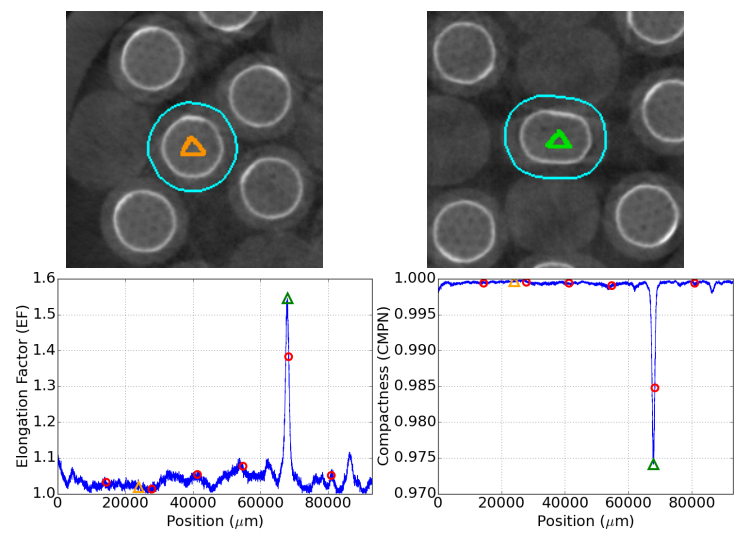

Figure 8: Validation of the strand section compression indicators on the N25 cable. Orange and green triangles are resp. normal and compressed locations along the same strand. On top row, the sections contours are superimposed on source images. Red circles are locations where partial scans have been registered.

tions and on Rutherford cables.

\subsubsection{Clustering}

Estimate the quality of the clustering w.r.t. ground truths is an important issue. However, no ground truths are currently available. Due to a large number of strands and an important number of constraints to satisfy, their construction is indeed a difficult task, especially when the cables are very short (as here). Since the calculus of twist-pitches requires the cables to be clustered, we believe that obtaining reasonable, close-to-expected values, verifies the clusterings.

\subsection{Results}

\subsubsection{Cables-in-conduit}

First, we have compared the values of void fraction obtained experimentally to nominal values. The result of these comparisons is presented in Tab. 4. In average, the void fraction differs (in absolute value) by $1.23 \%$ and does not exceed $2.72 \%$. Compared to the cable N05, it also confirms that the cables N22 and N25 are more likely do develop damages due to larger void fraction.

Similarly, experimental values of twist-pitches have been compared to nominal values. Depending of the length of the sample, more than one or less than one twisting periods can be available. For the studied cables, an exhaustive comparison to references values is possible. The result of these comparisons is shown in Tab. 5. In average, the twist-pitches differ (in absolute value) 
Table 3: Accuracy of the segmentation procedures with respect to ground truths for several metrics. The keyword "all" gathers the contours of s.c. and n.s.c. strands as well as the contours of the conduit (if need be).

\begin{tabular}{|c|c|c|c|c|c|c|c|}
\cline { 2 - 7 } \multicolumn{2}{c|}{} & DC (\%) & VOE (\%) & RVD (\%) & RMSD (pixels) & MD (pixels) & CD (pixels) \\
\hline \multirow{4}{*}{ In-conduit } & s.c. & $95.77 \pm 1.04$ & $8.11 \pm 1.91$ & $-7.47 \pm 2.17$ & $1.60 \pm 0.28$ & $3.73 \pm 0.97$ & $0.59 \pm 0.36$ \\
\cline { 2 - 8 } & n.s.c. & $95.47 \pm 1.31$ & $8.63 \pm 2.35$ & $-3.04 \pm 2.89$ & $1.85 \pm 0.41$ & $5.04 \pm 1.63$ & $1.22 \pm 0.84$ \\
\cline { 2 - 8 } & s.c. + n.s.c. & $95.63 \pm 1.18$ & $8.35 \pm 2.14$ & $-5.44 \pm 3.35$ & $1.72 \pm 0.37$ & $4.33 \pm 1.47$ & $0.88 \pm 0.70$ \\
\cline { 2 - 8 } & Conduit & $99.67 \pm 0.06$ & $0.67 \pm 0.12$ & $-0.07 \pm 0.23$ & $1.69 \pm 0.17$ & $5.16 \pm 1.15$ & $/$ \\
\cline { 2 - 8 } Power & all & $95.72 \pm 1.31$ & $8.18 \pm 2.39$ & $-5.33 \pm 3.41$ & $1.71 \pm 0.36$ & $4.34 \pm 1.47$ & $/$ \\
\cline { 2 - 8 } & s.c. & $97.86 \pm 0.51$ & $4.18 \pm 0.97$ & $1.06 \pm 1.69$ & $1.62 \pm 0.21$ & $4.54 \pm 1.03$ & $0.86 \pm 0.48$ \\
\cline { 2 - 8 } & n.s.c. & $97.99 \pm 0.44$ & $3.93 \pm 0.84$ & $-0.87 \pm 1.53$ & $1.57 \pm 0.23$ & $4.27 \pm 1.02$ & $0.83 \pm 0.43$ \\
\cline { 2 - 8 } & S.c. + n.s.c. & $97.94 \pm 0.47$ & $4.03 \pm 0.90$ & $-0.11 \pm 1.85$ & $1.59 \pm 0.22$ & $4.38 \pm 1.03$ & $0.84 \pm 0.45$ \\
\cline { 2 - 8 } & Conduit & $99.80 \pm 0.10$ & $0.39 \pm 0.20$ & $0.38 \pm 0.22$ & $2.10 \pm 0.35$ & $7.06 \pm 3.43$ & $/$ \\
\hline \hline Rutherford & all & $97.97 \pm 0.52$ & $3.97 \pm 1.01$ & $-0.10 \pm 1.84$ & $1.60 \pm 0.23$ & $4.42 \pm 1.16$ & $/$ \\
\hline
\end{tabular}

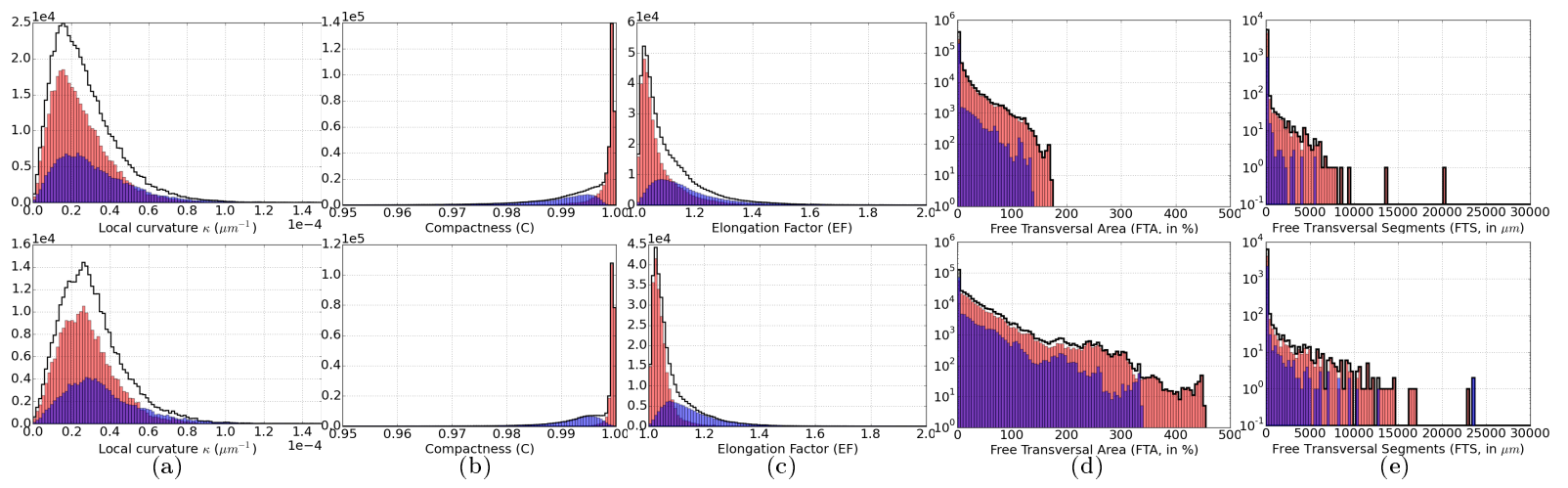

Figure 9: Distributions of damage indicators for s.c. (pink), n.s.c. (blue) and both (black thick line) strands along the cables-in-conduit N05 (top row) and N25 (bottom row). The y-axis of FTA and FTS is in log-scale.

by $2.93 \mathrm{~mm}(3.25 \%)$ and do not exceed $9.38 \mathrm{~mm}$ (7.5\%). Despite the randomness injected in these cables, this result demonstrates that twist-pitches remain close to nominal values.

Additionally, we have computed the correlations between local curvature and strand sections compression indicators. Then, we have decided to retain the most relevant couples of indicators for which the absolute value of the correlation coefficient is larger than 0.8. Not surprisingly, EF and CMPN are well correlated. But most importantly, no correlation have been identified between locally elevated curvature and other indicators, meaning that bendings and compressions locations do not coincide.

Moreover, we have compared in Fig. 9 the distributions of several damage indicators (local curvature, CMPN, EF, FTA and FTS) of two cables-in-conduit that present different lengths, void fractions and twist-pitches: N05 (top row) and N25 (bottom row). The distribution of FTS is obtained by thresholding FTA at $15 \%$, which appears to be a good value to keep significant transversal moves. For each image, we provide both the distribution over s.c. (pink), n.s.c. (blue) and both (black thick line) strands. For both cables, local curvatures remain very small due to the near linearity of strands. Also, the shape of the related distribution suggests that it is centered around the curvature induced by the twist-pitch and that local curvature maxima correspond to locations of potential conductivity loss. The mean of these distributions is nearly the same $\left(2 \times 10^{-5} \mu \mathrm{m}^{-1}\right)$ corresponding to a curvature radius of $50 \mathrm{~mm}$. Nevertheless, the mean is slightly larger for the N25 cable. This is consistent with the fact that this cable has smaller twist-pitch values. Additionally, the distribution of EF and CMPN on both cables is centered, as expected, on a value close to one, meaning that strands sections are mainly circular. The higher peak and longer tail of the EF distribution on s.c. strands for N05 than for N25 indicates more frequent and more heavily compressed strands. This is expected as a consequence of stronger compaction of the N05 cable.

Finally, the tail of the FTA and FTS distributions of the N05 cable is shorter than for the N25 cable. This means that the strands of the N05 cable have much less space to move and that the 
Table 4: Comparison of experimental and nominal values of void fraction for all cables-in-conduit (top) and all power cables (bottom).

\begin{tabular}{|c|c|c|}
\cline { 2 - 3 } \multicolumn{1}{c|}{} & $\begin{array}{c}\text { Measured void } \\
\text { fraction (\%) }\end{array}$ & $\begin{array}{c}\text { Nominal void } \\
\text { fraction (\%) }\end{array}$ \\
\hline N05 & $24.16 \pm 0.19$ & $25 \%$ \\
\hline N22 & $30.28 \pm 0.16$ & $33 \%$ \\
\hline \hline N25 & $33.13 \pm 0.14$ & $33 \%$ \\
\hline mgb2_113 & $27.57 \pm 0.38$ & $24.7 \%$ \\
\hline
\end{tabular}

Table 5: Comparison of experimental and nominal values of twist-pitches for all cables-in-conduit.

\begin{tabular}{|c|c|c|c|c|}
\hline Cable & Stage & $\begin{array}{l}\text { Twisting } \\
\text { Period }\end{array}$ & $\begin{array}{l}\text { Experimental } \\
\text { twist-pitches } \\
(\mathrm{mm})\end{array}$ & $\begin{array}{c}\text { Nominal } \\
\text { twist-pitches } \\
(\mathrm{mm})\end{array}$ \\
\hline \multirow{5}{*}{ N05 } & \multirow{3}{*}{1} & 1 & $43.81 \pm 1.46$ & 45 \\
\hline & & 2 & $87.95 \pm 2.52$ & 90 \\
\hline & & 3 & $131.57 \pm 5.89$ & 135 \\
\hline & 2 & 1 & $83.10 \pm 6.00$ & 85 \\
\hline & 3 & 1 & $129.06 \pm 0.00$ & 125 \\
\hline \multirow{5}{*}{$\mathrm{N} 22$} & \multirow{3}{*}{1} & 1 & $43.92 \pm 1.26$ & 45 \\
\hline & & 2 & $87.82 \pm 2.24$ & 90 \\
\hline & & 3 & $131.77 \pm 3.63$ & 135 \\
\hline & 2 & 1 & $82.22 \pm 2.80$ & 85 \\
\hline & 3 & 1 & $115.62 \pm 0.00$ & 125 \\
\hline \multirow{3}{*}{ N25 } & \multirow{2}{*}{1} & 1 & $35.84 \pm 1.13$ & 35 \\
\hline & & 2 & $72.93 \pm 2.48$ & 70 \\
\hline & 2 & 1 & $61.89 \pm 1.49$ & 65 \\
\hline
\end{tabular}

segments where they can move are shorter. These distributions tend to decrease, meaning that a large area where a strand can move is less likely to occur in the cable than a small one. Again, this is an expected consequence of the stronger compaction of the N05 cable. Moreover, large areas are more likely to occur for s.c. strands than for n.s.c. strands. This is consistent with the fact that the number s.c. strands is greater than the number of n.s.c. strands (see Table 1).

\subsubsection{Power cables}

As for cables-in-conduit, the values of void fraction obtained experimentally from segmentations have been first compared to nominal values. The result of these comparisons is available in Tab. 4 . In average, the void fraction differs (in absolute value) by $2.6 \%$ from the nominal values and does not exceed $2.87 \%$ (which appears to be slightly larger than for cables-in-conduit).

Finally, we have compared in Fig. 10 the distributions of the local curvature of the two power cables: mgb2_113 and mgb2_133. As for cablesin-conduit, the strands have near linear trajecto-
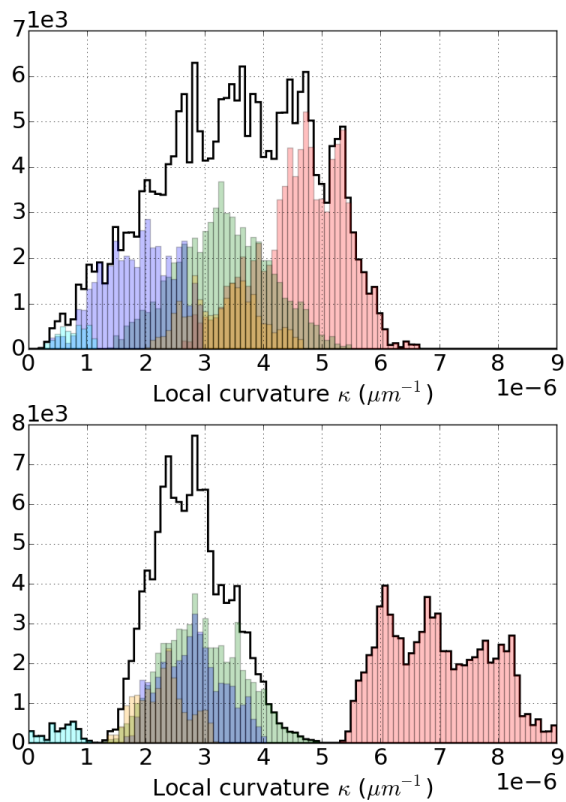

Figure 10: Distributions of the local curvature $\kappa$ along the power cables mgb2_113 (top) and mgb2_133 (bottom) for s.c. strands (pink), n.s.c. strands (green, blue, orange, cyan, resp. from outer to inner stages) and all strands (black thick line).

ries, thus leading to small values of local curvature up to $9 \times 10^{-6} \mu \mathrm{m}^{-1}$. This corresponds to a maximum radius of curvature of $111 \mathrm{~mm}$. Unlike cables-in-conduit, the shape of the related distributions is multimodal where each mode is centered on the local curvature of the corresponding concentric layer of strands. Each mode appears to be well separated from the others. Whereas we expect to get an increasing local curvature as the concentric layer becomes large, it seems to be only partially true. A possible explanation is the existence of slightly different twist-pitches among these layers.

\subsubsection{Rutherford cables}

In Fig. 11, we have compared the distributions of several damage indicators (local curvature, CMPN and EF) of two Rutherford cables presenting a different length and number of strands: 18RRPN01 and 40RRPR02. Notice that the yaxis of all images is in log-scale to ease the analysis of results. In contrast to the other types of cables studied, the local curvature appears to be much larger (due to the folding of the cable), up to $2.75 \times 10^{-4} \mu \mathrm{m}^{-1}$. This corresponds to a radius of curvature of $3.63 \mathrm{~mm}$. Moreover, the distribution of all indicators share the same shape. More precisely, the head of these distributions corre- 

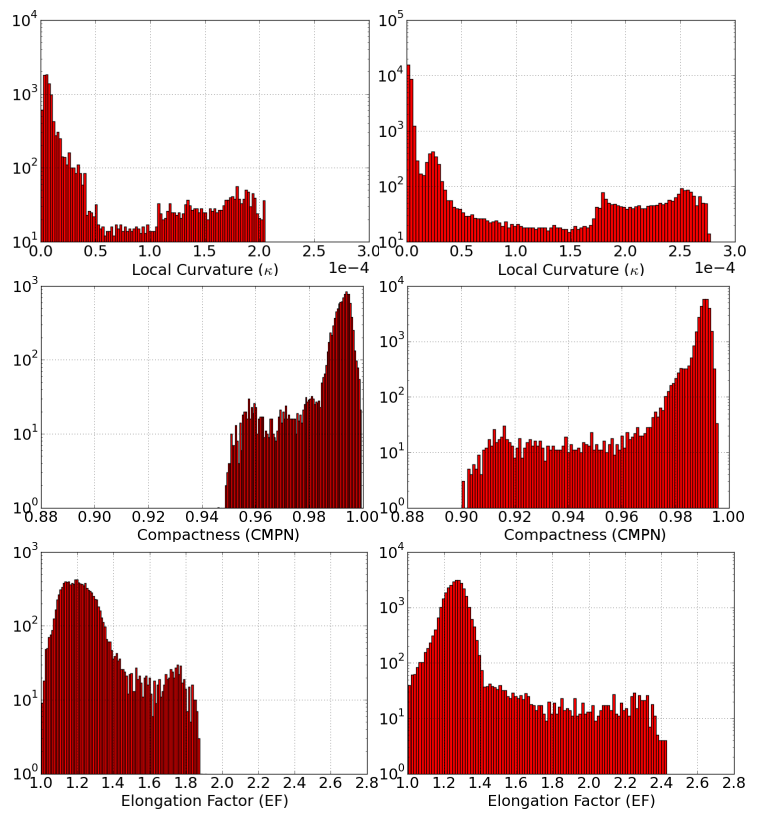

Figure 11: Distributions of the local curvature (top row), CMPN (middle row) and the elongation factor (bottom row) along the Rutherford cables 18RRPN01 and 40RRPR02. For the purpose of visualization, the $\mathrm{y}$-axis of all images is in log-scale.

sponds to the circular and straight strands with null values of local curvature and values close to one for the other indicators. The tail of these distributions corresponds to compressed and curved strands, on the sides of the cable.

Finally, the correlations between all the above indicators have been computed. The most relevant couples of indicators were selected as those having an absolute correlation coefficient above 0.8 . These correlations have permitted to conclude that EF and CMPN are the most correlated ones. The obtained correlations between local curvature and strand sections compression indicators also confirmed that bendings and compressions locations coincide quite well with a coefficient of about 0.7 in absolute value.

\section{CONCLUSION}

This paper presents a segmentation and geometry analysis of three types of cables and is, to our knowledge, the first one to provide methods and results to this aim. The segmentation results exhibit a good accuracy w.r.t. manually provided ground truth. The features appear to be relevant for detecting deformations such as crushing or bending. The results are now going to be analyzed. We have found, for instance, that s.c. and n.s.c. strands exhibit different distributions of the crushing-related features. Similarly, no spatial correlation between crushing and bending was found. A further statistical analysis of the results will be done in the future.

\section{REFERENCES}

Dice, L. (1945). Measures of the amount of ecologic association between species. Ecology, 26(3):297302.

Ginneken, B., Heimann, T., and Styner, M. (2007). $3 \mathrm{D}$ segmentation in the clinic: A grand challenge. In MICCAI Workshop.

IEC (2004). Âmes des câbles isolés. IEC Central Office Geneva Switzerland. CEI 60228:2004.

Ikonen, L. (2005). Pixel queue algorithm for geodesic distance transforms. Discrete Geometry for Computer Imagery, 3429:228-239.

Manil, P., Mouzouri, M., and Nunio, F. (2012). Mechanical modeling of low temperature superconducting cables at the strand level. IEEE Transactions on Applied Superconductivity, 22(3).

Meyer, F. (1991). Un algorithme optimal de ligne de partage des eaux. In Congrès AFCET-RFIA, volume 2, pages $847-859$.

Milanese, A., Devaux, M., Durante, M., Manil, P., Perez, J., Rifflet, J., De Rijk, G., and Rondeaux, F. (2012). Design of the EuCARD high field model dipole magnet FRESCA2. IEEE Transactions on Applied Superconductivity, 22(3).

Mittelmann, H. (2007). Recent benchmarks of optimization software. In European Conference on Operational Research.

Montero, R. (2009). State of the art of compactness and circularity measures. International Mathematical Forum, 4(27):1305-1335.

Oberli, L. (2013). Development of the $\mathrm{Nb}_{3} \mathrm{Sn}$ Rutherford cable for the EuCARD high field dipole magnet FRESCA2. IEEE Transactions on Applied Superconductivity, 23(3).

Seidel, M. and Sturge, D. (2009). Tensile surface and structure: A pratical guide to cable and membrane construction. Wiley.

Sternberg, R. (1986). Grayscale morphology. Computer Vision Graphics and Image Processing, 35(3):333-355.

Torre, A., Bajas, H., and Ciazynski, D. (2014). Mechanical and electrical modeling of strands in two ITER CS cable designs. IEEE Transactions on Applied Superconductivity, 24(3).

Žunić, J. (2012). Shape descriptors for image analysis. Zbornik Radova, 15(23):5-38.

Weiss, K., Heller, R., Fietz, W., Duchateau, J., Dolgetta, N., and Vostner, A. (2007). Systematic approach to examine the strain effect on the critical current of $\mathrm{Nb}_{3} \mathrm{Sn}$ cable-in-conduitconductors. IEEE Transactions on Applied Superconductivity, 17(2):1469-1472. 